\title{
Design and Simulation Analysis for Integrated Vehicle Chassis-Network Control System Based on CAN Network
}

\author{
Wei Yu and Ning Sun \\ College of Automobile and Transport Engineering, Nanjing Forestry University, Nanjing 210037, China \\ Correspondence should be addressed to Wei Yu; yuwei505@163.com
}

Received 30 March 2016; Revised 18 May 2016; Accepted 26 June 2016

Academic Editor: Antonio Fernández-Caballero

Copyright (C) 2016 W. Yu and N. Sun. This is an open access article distributed under the Creative Commons Attribution License, which permits unrestricted use, distribution, and reproduction in any medium, provided the original work is properly cited.

\begin{abstract}
Due to the different functions of the system used in the vehicle chassis control, the hierarchical control strategy also leads to many kinds of the network topology structure. According to the hierarchical control principle, this research puts forward the integrated control strategy of the chassis based on supervision mechanism. The purpose is to consider how the integrated control architecture affects the control performance of the system after the intervention of CAN network. Based on the principle of hierarchical control and fuzzy control, a fuzzy controller is designed, which is used to monitor and coordinate the ESP, AFS, and ARS. And the IVC system is constructed with the upper supervisory controller and three subcontrol systems on the Simulink platform. The network topology structure of IVC is proposed, and the IVC communication matrix based on CAN network communication is designed. With the common sensors and the subcontrollers as the CAN network independent nodes, the network induced delay and packet loss rate on the system control performance are studied by simulation. The results show that the simulation method can be used for designing the communication network of the vehicle.
\end{abstract}

\section{Introduction}

From the current development of the control system of vehicle chassis, integration and networking trend is very obvious [1]. The architecture of system control and network has different degrees of influence on the stability of chassis control. Due to the different functions of the system used in the vehicle chassis control, the hierarchical control strategy also leads to many kinds of the network topology structure and the distribution of the system computing tasks. In the 80 s of last century, the researchers began to decompose the complex chassis control problem into a number of subcontrol systems and then use a mechanism to coordinate the dynamic relationship between the subsystems to meet the control requirements. Therefore, the research and discussion of the integrated control architecture of the chassis form [2-9] began to become the focus.

As far as the integrated control strategy of vehicle chassis is concerned, numerous studies have shown that the hierarchical control can effectively reduce the operation conflict between different functional subsystems, and quickly and effectively make the vehicle get the best performance. A large number of literatures [2-4] divide chassis control into different subcontrol systems according to the vertical, lateral, and normal control systems, and the integrated optimization control of the chassis is realized through the hierarchical control strategy. Li et al. put forward the integrated control structure of chassis based on the combination of the main loop and servo loop and discussed the problems of different directional force and force distribution of the chassis [6].

Chang and Gordon divided the chassis control system into three layers to achieve the active collision avoidance control [8]. Using the system architecture for the independent control units of the chassis of integrated control with upper coordinated control [10] can effectively adjust the collaborative work of control units, avoid the conflict of the controllers, and make the vehicle obtain the best running state. Through the analysis of the complex working conditions, the supervision mechanism is used to coordinate the multiple control 
systems of the vehicle chassis, which can achieve a very good control effect of the system integration [11].

For these reasons, this paper firstly according to the hierarchical control principle, puts forward integrated control strategy of the chassis based on supervision mechanism. Based on the verification of the validity of this control strategy, the purpose of the study is to consider how the integrated control architecture affects the control performance of the system after the intervention of CAN network. Some exploratory simulation research is carried out. In order to facilitate the discussion, the integrated control system of network of the vehicle chassis based on network communication is abbreviated as IVC-NCS, namely, Integrated Vehicle Chassis-Network Control System.

\section{Dynamic Model of the Vehicle}

At present, international vehicle coordinate system mainly has two kinds [12]: one is SAE vehicle coordinate system issued by American Society of Automotive Engineers and another one is ISO vehicle coordinate system issued by International Standardization Organization. In this paper, SAE vehicle coordinate system is used for modeling, calculation, and analysis of vehicle dynamics. Based on the above assumptions, the nonlinear vehicle dynamics model has eight degrees of freedom.

There are a lot of tire models to calculate the complex nonlinear force between the road surface and the wheel. The most commonly used in the project is magic formula raised by Pacejka of Holland $[13,14]$ and unified tire model of overall conditions raised by Guo Konhui of China [15]. This paper uses Dugoff tire model [16], which is often used in computer simulation. It belongs to analytical model, and the parameters are small and easy to obtain.

\section{Architecture of IVC-NCS Based on Supervision Mechanism}

Figure 1 shows the architecture of IVC-NCS based on supervision mechanism. Three subcontrol systems are ESP, AFS, and ARS. Each subsystem can be controlled according to the calculation of local state variables. Based on the global state of the vehicle, the upper supervision controller judges the function weight by the vehicle stability for each subcontrol systems. The implementation of the execution mechanism is determined by the calculation results of each subcontroller and control weight.

3.1. ESP Subcontrol System. ESP takes the handling stability as the control target on the critical conditions of the whole vehicle. By controlling the braking intensity of four wheels, the electronic control of vehicle active safety is finished. The yaw rate tracking is the control target by applying a braking force at the right wheel to correct the unstable state of the vehicle. The system adopts sliding mode control strategy, and the tracking error of yaw rate is defined as sliding mode variable:

$$
s_{b}=r-r_{\mathrm{idl}},
$$

where $r$ is the actual yaw rate and $r_{\text {idl }}$ is the ideal yaw rate.

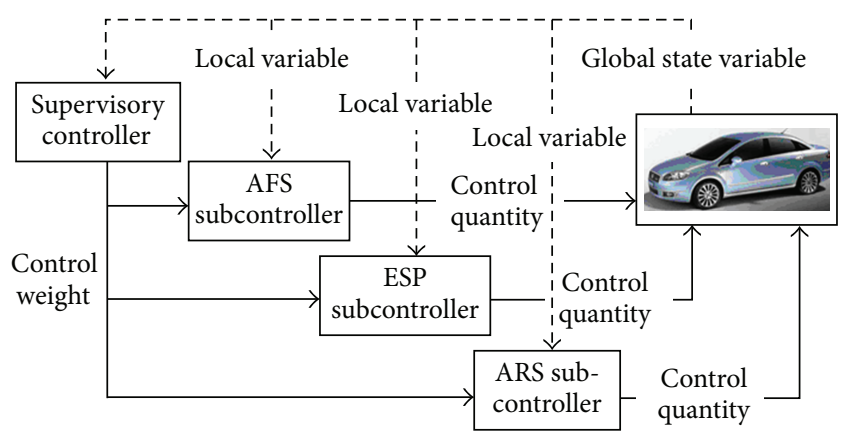

FIGURE 1: Architecture of IVC-NCS based on supervision mechanism.

The condition for reaching the sliding surface is defined as

$$
\dot{s}_{b}=-\lambda_{b} s_{b}-\kappa_{b} \operatorname{sat}\left(\frac{s_{b}}{\varepsilon_{b}}\right),
$$

where $\lambda_{b}$ and $\kappa_{b}$ are all positive constants, $\lambda_{b}$ reflects the response speed of yaw tracking controller, $\kappa_{b}$ shows the convergence rate of sliding mode surface of the system, $S_{b}$ is the tracking error of yaw rate, and $\varepsilon_{b}$ is the thickness of boundary layer.

The sliding mode controller satisfies the stability condition of Lyapunov sense.

Ignoring the inclination of vehicle and considering formula (2), when ESP control system acts on braking of single vehicle, the calculation formula of additional yaw rate torque is gotten:

$$
\begin{aligned}
\frac{M_{z c}}{I_{z z}}= & -\frac{L_{f}\left(F_{y \mathrm{fl}}+F_{y \mathrm{fr}}\right)-L_{r}\left(F_{y \mathrm{rl}}+F_{y \mathrm{rr}}\right)}{I_{z z}}+\dot{r}_{\mathrm{idl}} \\
& -\lambda_{b} s_{b}-\kappa_{b} \operatorname{sat}\left(\frac{s_{b}}{\varepsilon_{b}}\right),
\end{aligned}
$$

where $M_{z c}$ is the additional yaw torque generated by longitudinal driving force or braking force, $I_{z z}$ is the moment of inertia of the vehicle body around $z$-axis, $L_{f}$ is the vertical distance from the centroid to the front axle, $L_{r}$ is the vertical distance from the centroid to the rear axle, $F_{y \mathrm{fl}}$ is the longitudinal force of the ground on the left front tire, $F_{y \mathrm{fr}}$ is the longitudinal force of the ground on the right front tire, $F_{y \mathrm{rl}}$ is the longitudinal force of the ground on the left rear tire, $F_{y \mathrm{rr}}$ is the longitudinal force of the ground on the right rear tire, $r_{\text {idl }}$ is the ideal yaw rate, $\lambda_{b}$ reflects the response speed of yaw tracking controller, $\kappa_{b}$ shows the convergence rate of sliding mode surface of the system, $S_{b}$ is the tracking error of yaw rate, and $\varepsilon_{b}$ is the thickness of boundary layer.

In order to improve the unstable state in extreme conditions, braking force is applied to inward rear wheel when the vehicle has the understeer, or braking force is applied to outward front wheel when the vehicle has the oversteer. It can quickly and effectively improve vehicle stability. Therefore, the additional yaw torque calculated by formula (3) is converted to the equivalent braking force that can be applied to wheel with the most effective braking force. 
3.2. AFS Subcontrol System. In steering system of the vehicle chassis, a relatively independent subcontrol system such as AFS is increased to adjust the front wheel angle for obtaining the optimum performance of IVC-NCS.

The system adopts sliding mode control strategy, and the tracking error of yaw rate is defined as sliding mode variable:

$$
s_{s f}=r-r_{\mathrm{idl}},
$$

where $r$ is the actual yaw rate and $r_{\text {idl }}$ is the ideal yaw rate.

The condition for reaching the sliding surface is defined as

$$
\dot{s}_{s f}=-\lambda_{s f} s_{s f}-\kappa_{s f} \operatorname{sat}\left(\frac{s_{s f}}{\varepsilon_{s f}}\right) \text {, }
$$

where

$$
\operatorname{sat}\left(\frac{s_{s f}}{\varepsilon_{s f}}\right)= \begin{cases}\frac{s_{s f}}{\varepsilon_{s f}}, & \left|s_{s f}\right|<\varepsilon_{s f} \\ \operatorname{sign}\left(\frac{s_{s f}}{\varepsilon_{s f}}\right), & \left|s_{s f}\right| \geq \varepsilon_{s f},\end{cases}
$$

where $\lambda_{s f}$ and $\kappa_{s f}$ are all positive constants, $\lambda_{s f}$ reflects the response speed of yaw tracking controller, $\kappa_{s f}$ shows the convergence rate of sliding mode surface of the system, $s_{s f}$ is the tracking error of yaw rate, and $\varepsilon_{s f}$ is the thickness of boundary layer.

The control law of steering angle about front wheel is

$$
\begin{aligned}
\delta_{f}= & \frac{1}{b_{21}}\left[-a_{21} V_{y}-a_{22} r+\dot{r}_{\mathrm{idl}}-\lambda_{f}\left(r-r_{\mathrm{idl}}\right)\right] \\
& -\kappa_{f} \operatorname{sat}\left(\frac{s_{s f}}{\varepsilon_{s f}}\right),
\end{aligned}
$$

where $V_{y}$ is the lateral vehicle speed, $r$ is the actual yaw rate, $r_{\text {idl }}$ is the ideal yaw rate, $\lambda_{f}$ reflects the response speed of yaw tracking controller, $\kappa_{f}$ shows the convergence rate of sliding mode surface of the system, $s_{s f}$ is the tracking error of yaw rate, and $\varepsilon_{s f}$ is the thickness of boundary layer.

According to the vehicle model of two degree of freedom, $b_{21}=2 L_{f} C_{f} / I_{z z}$, where $L_{f}$ is the vertical distance from the centroid to the front axle, $C_{f}$ is the pitch damping, $I_{z z}$ is the moment of inertia of the vehicle body around $Z$ axis.

And $a_{21}=\left(2 L_{r} C_{r}-2 L_{f} C_{f}\right) / I_{z z} V_{x}$, where $L_{r}$ is the vertical distance from the centroid to the rear axle, $C_{r}$ is the caster damping, $L_{f}$ is the vertical distance from the centroid to the front axle, $I_{z z}$ is the moment of inertia of the vehicle body around $z$-axis, $V_{x}$ is the longitudinal speed.

And $a_{22}=-\left(2 L_{f}^{2} C_{f}+2 L_{r}^{2} C_{r}\right) / I_{z z} V_{x}$, where $L_{f}$ is the vertical distance from the centroid to the front axle, $C_{f}$ is the pitch damping, $L_{r}$ is the vertical distance from the centroid to the rear axle, $C_{r}$ is the caster damping, $I_{z z}$ is the moment of inertia of the vehicle body around $z$-axis, and $V_{x}$ is the longitudinal speed.

3.3. ARS Subcontrol System. Active four-wheel steering technology can improve the handling stability of the vehicle at high speed and the controlling flexibility at low speed. The ideal yaw rate calculated by vehicle model of two degrees of freedom is the tracked target. So ARS takes round steering angle as the controlled variable.

The system adopts sliding mode control strategy, and the tracking error of yaw rate is defined as sliding mode variable:

$$
s_{s r}=r-r_{\text {idl }}
$$

where $r$ is the actual yaw rate and $r_{\text {idl }}$ is the ideal yaw rate.

The condition for reaching the sliding surface is defined as

$$
\dot{s}_{s r}=-\lambda_{s r} s_{s r}-\kappa_{s r} \operatorname{sat}\left(\frac{s_{s r}}{\varepsilon_{s r}}\right),
$$

where

$$
\operatorname{sat}\left(\frac{s_{s r}}{\varepsilon_{s r}}\right)= \begin{cases}\frac{s_{s r}}{\varepsilon_{s r}}, & \left|s_{s r}\right|<\varepsilon_{s r} \\ \operatorname{sign}\left(\frac{s_{s r}}{\varepsilon_{s r}}\right), & \left|s_{s r}\right| \geq \varepsilon_{s r},\end{cases}
$$

where $\lambda_{s r}$ and $\kappa_{s r}$ are all positive constants, $\lambda_{s r}$ reflects the response speed of yaw tracking controller, $\kappa_{s r}$ shows the convergence rate of sliding mode surface of the system, $s_{s r}$ is the tracking error of yaw rate, and $\varepsilon_{s r}$ is the thickness of boundary layer.

In order to restrain the shake of high frequency caused by frequent switching on the sliding surface, $\varepsilon_{s r}$ is taken as the thickness of the boundary layer. $\lambda_{s r}$ reflects the response speed of yaw tracking controller, and $\kappa_{s r}$ reflects the rate how the system reaches the sliding surface.

3.4. Upper Supervisory Controller Design. The control idea of the supervisory controller is as follows: judging the steady state of the vehicle according to the stability factor, distributing the weight of the control function of three subcontrollers, and coordinating the output of each subcontroller.

Firstly, the stability factor of front and rear wheels is defined as [17]

$$
\mathrm{SF}_{f}=\left|c_{1} \alpha_{f}+c_{2} \dot{\alpha}_{f}\right|
$$

where $\mathrm{SF}_{f}$ is the possibility that the front wheels come into the slipping state and $\alpha_{f}$ is the corresponding sideslip angle of the middle of the left and right wheels on the front axle:

$$
\mathrm{SF}_{r}=\left|c_{1} \alpha_{r}+c_{2} \dot{\alpha}_{r}\right|
$$

where $\mathrm{SF}_{r}$ is the possibility that the rear wheels come into the slipping state and $\alpha_{r}$ is the corresponding sideslip angle of the middle of the left and right wheels on the rear axle.

$c_{1}$ and $c_{2}$ can be obtained by analyzing the relationship between the phase plane and the steering stability of the tire [18].

$\mathrm{SF}_{f}$ and $\mathrm{SF}_{r}$ show the possibility of the corresponding wheel beginning to side. The larger the value, the bigger the side slipping possibility of corresponding wheel, namely, the smaller the control margin provided by the wheel. Conversely, the smaller the value, the greater the effective strength of corresponding wheel. 
TABLE 1: Rules of fuzzy controller of IVC.

\begin{tabular}{lcccc}
\hline $\mathrm{SF}_{f}$ & $\mathrm{SF}_{r}$ & $W_{\mathrm{AFS}}$ & $W_{\mathrm{ARS}}$ & $W_{\mathrm{ESP}}$ \\
\hline $\mathrm{S}$ & $\mathrm{S}$ & $\mathrm{B}$ & $\mathrm{B}$ & $\mathrm{S}$ \\
$\mathrm{S}$ & $\mathrm{MS}$ & $\mathrm{B}$ & $\mathrm{M}$ & $\mathrm{S}$ \\
$\mathrm{S}$ & $\mathrm{MB}$ & $\mathrm{B}$ & $\mathrm{S}$ & $\mathrm{S}$ \\
$\mathrm{S}$ & $\mathrm{B}$ & $\mathrm{B}$ & $\mathrm{S}$ & $\mathrm{MS}$ \\
$\mathrm{MS}$ & $\mathrm{S}$ & $\mathrm{M}$ & $\mathrm{B}$ & $\mathrm{S}$ \\
$\mathrm{MS}$ & $\mathrm{MS}$ & $\mathrm{M}$ & $\mathrm{M}$ & $\mathrm{MS}$ \\
$\mathrm{MS}$ & $\mathrm{MB}$ & $\mathrm{M}$ & $\mathrm{S}$ & $\mathrm{MS}$ \\
$\mathrm{MS}$ & $\mathrm{B}$ & $\mathrm{M}$ & $\mathrm{S}$ & $\mathrm{MB}$ \\
$\mathrm{MB}$ & $\mathrm{S}$ & $\mathrm{M}$ & $\mathrm{B}$ & $\mathrm{S}$ \\
$\mathrm{MB}$ & $\mathrm{MS}$ & $\mathrm{M}$ & $\mathrm{M}$ & $\mathrm{S}$ \\
$\mathrm{MB}$ & $\mathrm{MB}$ & $\mathrm{M}$ & $\mathrm{M}$ & $\mathrm{MS}$ \\
$\mathrm{MB}$ & $\mathrm{B}$ & $\mathrm{S}$ & $\mathrm{S}$ & $\mathrm{MB}$ \\
$\mathrm{B}$ & $\mathrm{S}$ & $\mathrm{S}$ & $\mathrm{B}$ & $\mathrm{MS}$ \\
$\mathrm{B}$ & $\mathrm{MS}$ & $\mathrm{S}$ & $\mathrm{M}$ & $\mathrm{MS}$ \\
$\mathrm{B}$ & $\mathrm{MB}$ & $\mathrm{S}$ & $\mathrm{S}$ & $\mathrm{MB}$ \\
$\mathrm{B}$ & $\mathrm{B}$ & $\mathrm{S}$ & $\mathrm{S}$ & $\mathrm{B}$ \\
\hline
\end{tabular}

Through repeated simulation tests, when $\mathrm{SF}_{f}$ and $\mathrm{SF}_{r}$ are less than 0.7, the active steering control of the front and rear wheels can meet the requirements of vehicle stability. When $\mathrm{SF}_{f}$ or $\mathrm{SF}_{r}$ is bigger than 1.3, the use of ESP can be more effective to correct the excessive or lack steering state, which can keep the vehicle stable fast. When $\mathrm{SF}_{f}$ and $\mathrm{SF}_{r}$ are in the range from 0.7 to 1.3 , the wheels with smaller stability factor provide a greater role in vehicle stability control. Based on this, the design of fuzzy logic controller is designed as follows.

The controller takes the stability factors of the front and rare wheels, such as $\mathrm{SF}_{f}$ and $\mathrm{SF}_{r}$, as the input. The membership functions are in the same range $[0,2]$, and the fuzzy subset is $\{\mathrm{S}, \mathrm{MS}, \mathrm{MB}, \mathrm{B}\}$ as shown in Figure 2(a). The outputs of the controller are the control weights of three subcontrollers whose range is $[0,1]$.

The membership functions of AFS and ARS are the same, and fuzzy subset is $\{D, M, E\}$ as shown in Figure 2(b). The membership function of ESP subcontroller is shown in Figure $2(\mathrm{c})$, and fuzzy subset is $\{\mathrm{S}, \mathrm{MS}, \mathrm{MB}, \mathrm{B}\}$. The collection of letters is as follows: $\mathrm{S}$ is small, $\mathrm{M}$ is medium, and $\mathrm{B}$ is big.

Considering the actual application of the computation and real-time, all variables of the membership functions are easy to be calculated by the procedure, such as trigonometric function or trapezoidal function. Table 1 shows the inference rules of fuzzy controller of IVC.

\section{Network Topology Design of IVC-NCS}

According to system control strategy of IVC, combined with the control requirements of vehicle stability, the following several points are considered as the basis for the design. Actual limitations of vehicle space layout are as follows: because CAN network agreement and the corresponding international standards limit the length of the branches connecting the nodes and communication trunks, so network nodes in the actual space layout is one of the major considerations of network topology structure. Such as ARSC and
AFSC, they are divided into two control units to control the system separately, which is helpful to connect the sensors and the executing agency.

Load capacity constraint of network communication is as follows: for IVC-NCS, if all sensors, controllers, and actuators exist as independent network nodes and the network works in $250 \mathrm{Kbps}$ rate of regulated by vehicle high speed network of SAE, only from the theoretical calculation of CAN communication capability, its load capacity is difficult to meet the control requirements. While the communication speed is increased to $500 \mathrm{Kbps}$, the anti-interference ability of the node will be poor, so it is difficult to realize the high speed communication in the bad electromagnetic environment.

Real-time requirements of subsystems are as follows: three subsystems of IVC-NCS are the relatively independent closed-loop control system. ESP subsystem has higher request on real-time of wheel speed signals, which requires the executing agencies to react quickly according to control orders.

The sensors necessary for many systems are designed as independent network nodes. The subcontrol systems adopt traditional point-to-point connection in the controllers, sensors, and executing agencies. Its object is to obtain satisfactory real-time performance and reliability.

Based on above analysis, the network in Figure 3 is designed as IVC-NCS structure. CAN network is taken as the communication medium of the controller node, and each subsystem is connected with the traditional method of point to point. Considering that ESP system has obvious effect for vehicle stability in extreme conditions, the supervision and control tasks of the system and the control calculation of ESP are assigned to one node.

The sensor signals are the basis of the controller to judge the state of the vehicle and control instructions. When the network communication load suddenly increases, the probability of signal loss of low level sensors will be significantly increased. Therefore, in order to ensure the realtime performance of the sensor signal transmission, the message priority of the sensor nodes is set higher to avoid the message loss in the control cycle, which leads to control instability. Table 2 shows the communication matrix table of IVC-NCS. Messages Msg7 and Msg9, as the state messages of executing agencies, can help the controller nodes to understand the operation status of the system. Because they do not participate in the control calculation, so the priority is low, and the transmission cycle is relatively large.

\section{Simulation and Result Analysis}

According to nonlinear vehicle model with eight degrees of freedom to calculate the state of the vehicle, Simulink platform is used for simulation. Before the performance of IVC-NCS, the IVC system is simulated and tested to verify the effectiveness of the controller.

5.1. Effectiveness Verification of IVC System Control. In order to verify the effectiveness of IVC system, the sine curve and the step curve with the maximum value 5 degrees (about $0.087 \mathrm{rad}$ ) of the vehicle steering wheel are input to simulate 
TABLE 2: Communication matrix table of IVC-NCS.

\begin{tabular}{lcccccc}
\hline \multirow{2}{*}{ Message name } & $\begin{array}{c}\text { Message content } \\
\text { (signal) }\end{array}$ & Transmission node & \multicolumn{3}{c}{ Message property } & Signal description \\
\hline Msg1 & Interf & Node 1 & 1 & Pending & 8 bytes & Meaningless message \\
Msg2 & S_Yaw_Acc & Node 2 & 2 & $P=5 \mathrm{~ms}$ & 6 bytes & Yaw rate and lateral acceleration \\
Msg3 & S_StrWhAgl & Node 3 & 3 & $P=5 \mathrm{~ms}$ & 4 bytes & Steering wheel angle \\
Msg4 & S_Vx & Node 4 & 4 & $P=5 \mathrm{~ms}$ & 4 bytes & Longitudinal speed \\
Msg5 & Weight & Node 4 & 7 & AP & 4 bytes & Control weight of AFS and ARS \\
Msg6 & S_ARS & Node 5 & 5 & $P=5 \mathrm{~ms}$ & 8 bytes & Speed and rotation angle of rear wheel \\
Msg7 & D_ARS_M & Node 5 & 8 & $P=20 \mathrm{~ms}$ & 4 bytes & Rear wheel motor status \\
Msg8 & S_AFS & Node 6 & 6 & $P=5 \mathrm{~ms}$ & 8 bytes & Speed and rotation angle of front wheel \\
Msg9 & D_AFS_M & Node 6 & 9 & $P=20 \mathrm{~ms}$ & 4 bytes & Front wheel motor status \\
\hline
\end{tabular}

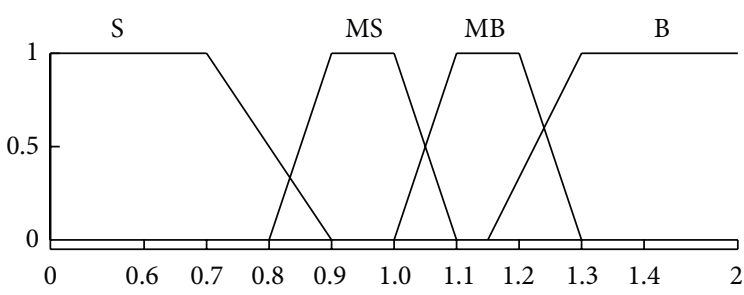

(a)

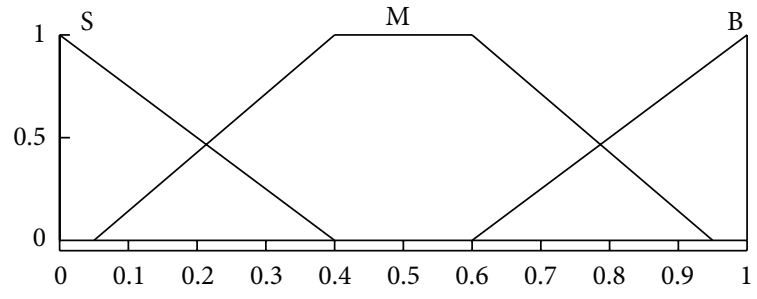

(b)

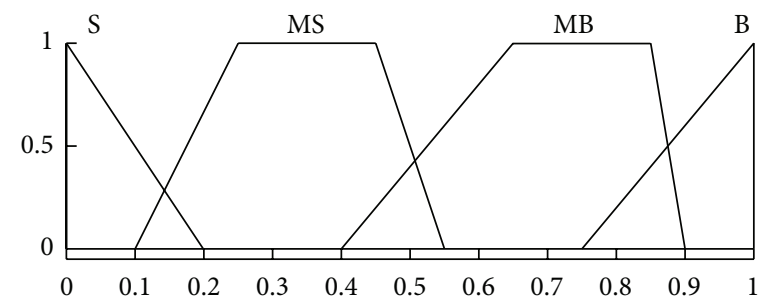

(c)

FIGURE 2: (a) $\mathrm{SF}_{f}$ and $\mathrm{SF}_{r}$. (b) Control weight $W_{\mathrm{AFS}}, W_{\mathrm{ARS}}$ of AFS and ARS. (c) Control weight $W_{\mathrm{ESP}}$ of ESP.

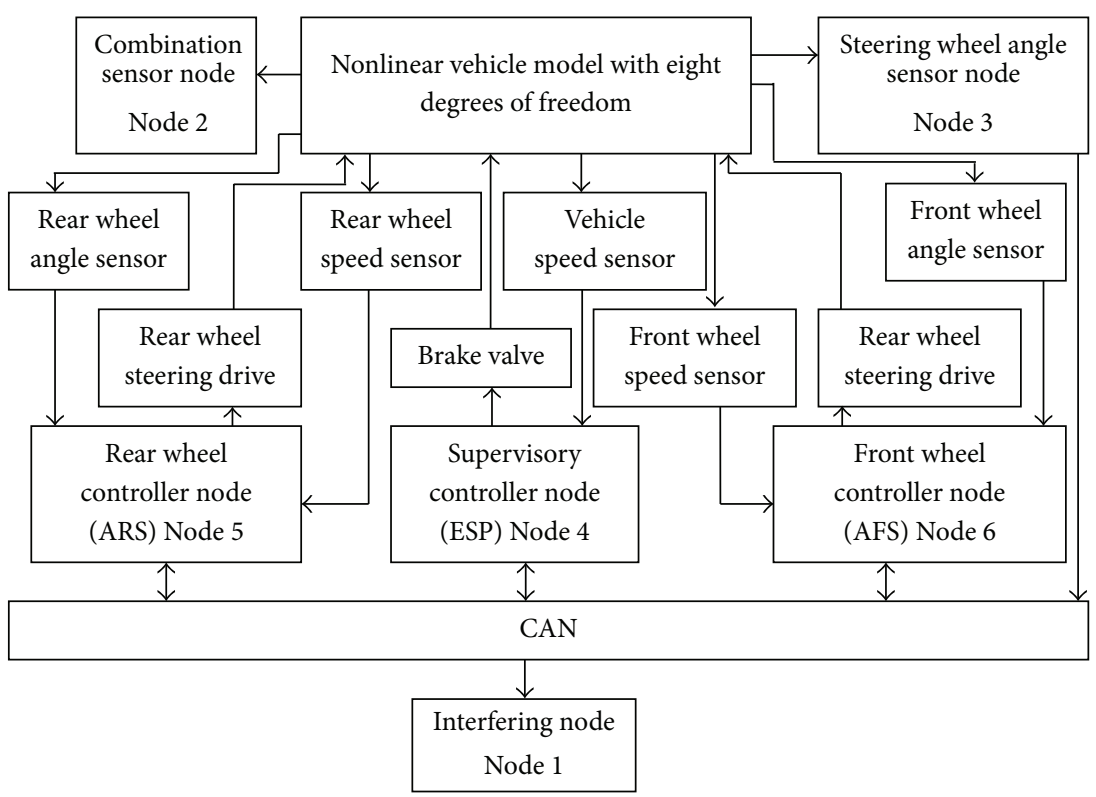

FIGURE 3: IVC-NCS network structure. 

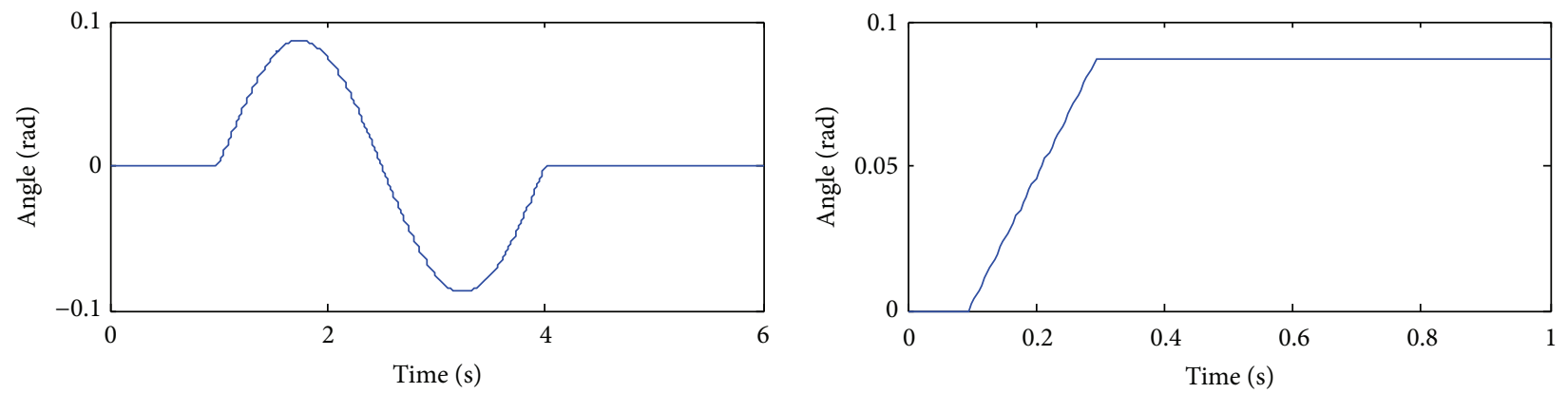

(a)

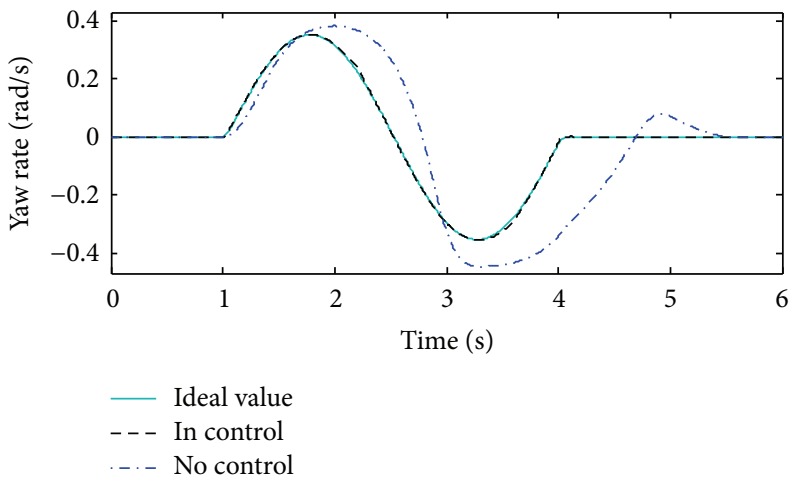

(b)

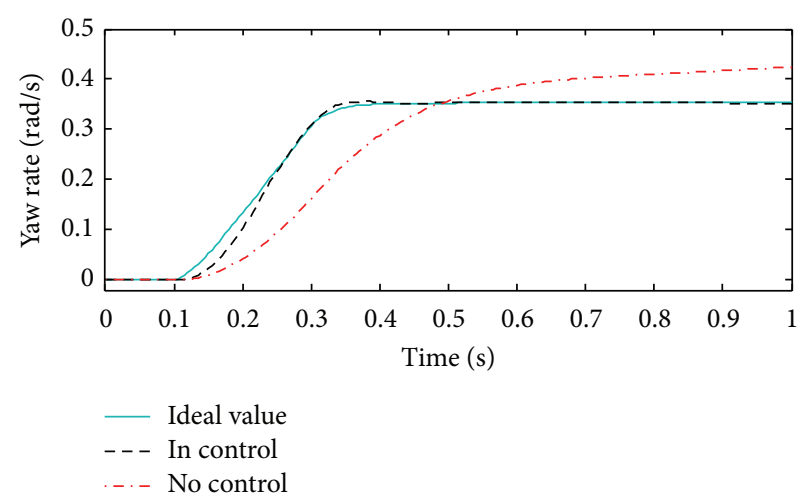

(c)

FiguRE 4: (a) Input curve of front wheel angle. (b) The response curve of yaw rate of the steering wheel with sine angle input. (c) The response curve of yaw rate of the steering wheel with step angle input.

the tracking response of the vehicle under different input yaw rates. According to the transmission ratio of the steering system, the corresponding input curve of front wheel steering angle is shown in Figure 4(a). The vehicle travels at a good road with a adhesion coefficient of 0.85 , and the initial speed is $25 \mathrm{~m} / \mathrm{s}$.

Figures 4(b) and 4(c) are the response curves of vehicle yaw rate at different angle inputs. It can be seen that the yaw rate of the controlled vehicle can quickly and effectively track the ideal value when compared with the system without the control. For the sine input, the execution of the vehicle is a nonstandard single lane change test. At this time due to the correction function of angle changes of the front wheel, so after the apparent slip, the yaw rate is settled in zero value, as shown in Figure 4(b).

Under the step input of steering wheel angle in Figure 4(c), the yaw rate of the vehicle without control cannot track the ideal value, which appears as the trend of divergence. So the vehicle cannot achieve stable circular motion and rollover because of instability. The yaw rate of the vehicle with controllers is good at tracking the ideal value. Simulation results show that the IVC system can effectively improve the stability vehicle in critical conditions, which verifies the effectiveness of the designed control system.

5.2. Simulation Analysis of IVC-NCS Based on CAN. In order to investigate the performance change of the designed IVC system after the CAN network is involved in the control, the stability of the vehicle was investigated using the same step input of the steering wheel. The initial speed is $25 \mathrm{~m} / \mathrm{s}$, and the road adhesion coefficient is 0.85 . Considering the practical application of CAN network with high speed, the communication rate is set to $250 \mathrm{Kbps}$. Node sends only data frames. If the interfering nodes do not send any message, the network load is about $84 \%$ when the maximum is filled. When the interference nodes send the interference message of high priority with $4 \mathrm{~ms}$ cycle, it can ensure that the network load is close to 1 but less than the network bandwidth, which ensures the system communication not to lose the frames.

According to the assumptions and simulation conditions, Figure 5 shows the comparison curve of yaw rate tracking about CAN network communication and point-to-point connection. Compared with point-to-point connection mode, the IVC system with CAN network connection can quickly and effectively track the ideal value under the condition of good network environment without changing the steady state of the control system. It can be clearly seen that, in the part of the amplified image, the network involves in the control system, which makes the yaw rate fluctuate with microamplitude. The overshoot of control increases from $3.1 \%$ of the point-to-point connection to $6 \%$ of the CAN network connection.

In order to investigate the influence of different network state on the control performance of the system, the tracking simulation test of vehicle yaw rate is carried out for different network load and packet loss rate. 


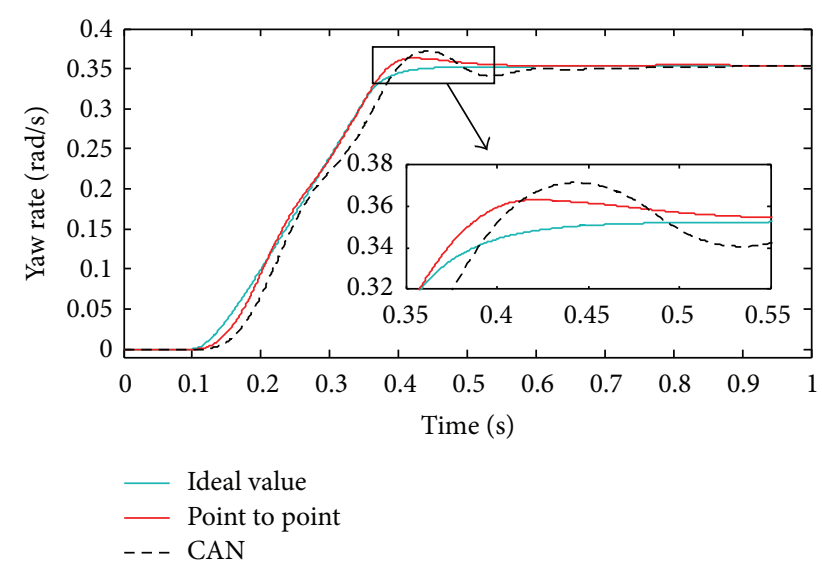

FIGURE 5: The response curve of IVC yaw rate of CAN network connection.

Figure 6 shows the response curve of different packet loss rates of IVC-NCS yaw rate. In the simulation process, the interference nodes do not send the messages. It can be seen that when the packet loss rate is lower than $20 \%$, the dynamic characteristic of the system becomes bad. In the packet loss rate of $5 \%$ and $20 \%$, the corresponding overshoots of the system are about $9 \%$ and $12.5 \%$. In $0.3 \mathrm{~s}$ after the step input of front wheel ends, the vehicle yaw rate can be stable to track the ideal value. When the packet loss rate is less than $40 \%$, the yaw rate of the vehicle can be finally stabilized at an ideal value. When the packet loss rate is more than $40 \%$, the yaw rate is obviously fluctuated in the ideal yaw rate tracking process. At $50 \%$, the overshoot of yaw rate increases rapidly to about $42 \%$, the vehicle begins to sideslip.

When the packet loss rate is up to $60 \%$, the vehicle yaw rate tracking is seriously lagging behind, which cannot achieve stable circular motion. The analysis shows that when the packet loss rate is low, the message transmission keep high success rate. The information of the sensors can be obtained by control nodes in time, so the controller works fast with little effect on the performance of system control. With the increase of packet loss rate, the control instructions cannot be timely generated and executed, which makes the control cycle become longer. The status of executing agency cannot be corrected in time. The input of executing agency will be too large or too small, which causes the control to fail.

Figure 7 shows that the interference nodes send the messages of highest priority in $4 \mathrm{~ms}$ cycle, and the network load is close to 1 . The long dashes are the response curve of yaw rate of CAN network without the interference, when the network load is about $84 \%$. The short dashed lines, dasheddotted lines, and bold dashed lines are separately response curves of yaw rate at $t$ different packet loss rates when the load is full.

Under the condition to meet the communication requirements of the control system, when the network load is close to 1 , the induced delay of the system is largest. It can be calculated, when the network load increases from $84 \%$ to nearly $100 \%$ and the overshoot increases from $6 \%$ to $7 \%$. When the network load is 1 and packet loss rate is $30 \%$,

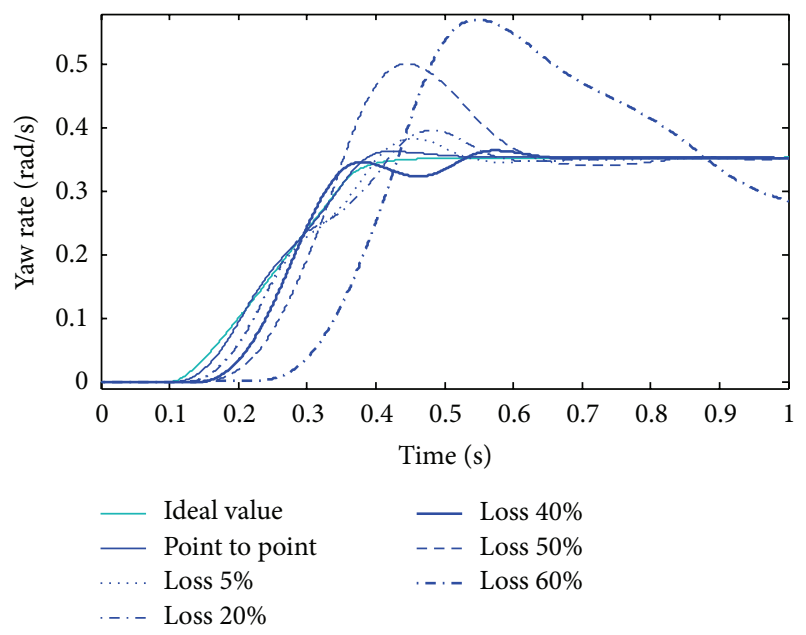

FIGURE 6: The response curve of different packet loss rates.

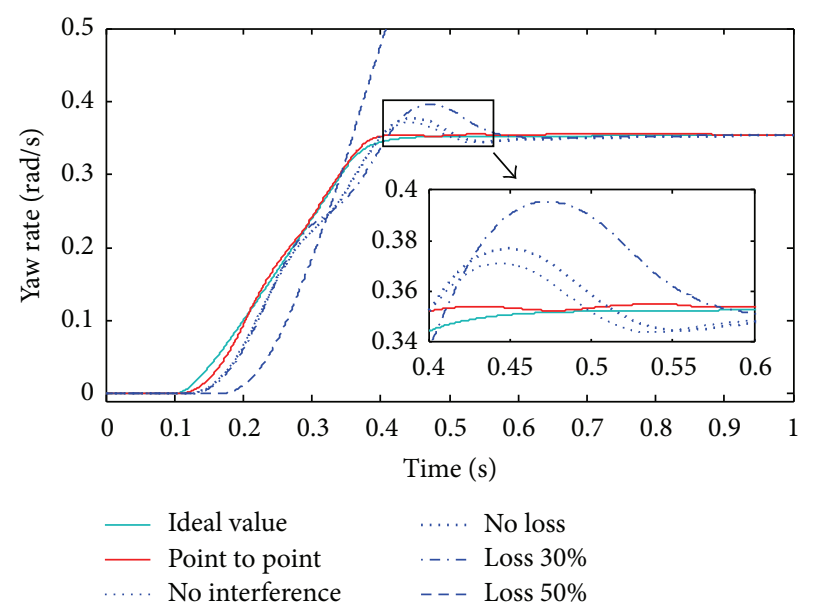

Figure 7: The response curve of yaw rate with full load of network communication.

the overshoot of yaw rate is $15.7 \%$. Therefore, although the network load increases, as long as network load can meet the communication requirements of the control system, the network intervention only has little effect on the quality of dynamic control, which does not change the steady characteristics of the system. The vehicle can achieve the stable circular motion within $0.3 \mathrm{~s}$ of the yaw rare input of the front wheel.

When the communication network is fully loaded and the packet loss rate is $50 \%$, the vehicle cannot complete the scheduled circular motion. The yaw rate of the vehicle diverges to make the vehicle out of control. The simulation results show that when the network bandwidth meets the needs of control system, the effect of the network induced delay of control system is very small and negligible. And the network packet loss will affect the performance of control system seriously. When the packet loss rate is up to $50 \%$, the system control performance will deteriorate significantly. 
5.3. Stability and Coordination Analysis. From the development of the vehicle chassis control system, the trend of integration and network is very obvious. The system control architecture and the network architecture form have different effects on the stability control of the chassis. In this paper, the design of the control system fully takes into account the stability of the chassis control performance.

Because ABS is the basis for the realization of ESP, and the latter needs to achieve the independent control of braking intensity about the four wheels, so ABS is designed as an independent four-channel mode. As one kind of the controller associated with safety and real-time, the execution and controller of ABS usually adopt directly connected manner, in order to reduce the information switching delay and ensure the safety and stability of the vehicle.

The control target of ESP system is to control the stability of the vehicle in the extreme conditions, through the control of braking strength of four wheels to achieve the active safety. In order to improve the unstable state of the vehicle in extreme conditions, applying the braking force on inward rear wheel with the understeer or on the outward front wheel with the oversteer can quickly and effectively improve the stability. Taking into account that ESP system has the obvious effect on the vehicle stability in extreme conditions, the study will assign the supervision and control tasks and the calculation of ESP control to one node.

For the performance of network control system, communication real-time performance is the most important factor affecting the control performance, which can be expressed and measured by network delay. The existence of network delay reduces the control performance of the system, which will lead to the loss of stability of the stable control system.

Especially, in extreme conditions, the change of the vehicle state is larger. When a large number of control instructions are lost, the adjustment of the new and old control instructions is bound to increase because of the large number of cycles, which will increase the action range of the actuator. Therefore, too much data packet loss is extremely unfavorable for the stability control. When the packet loss rate is less than a certain value, only the system dynamic characteristic becomes worse, and the system stability is not changed. When the packet loss rate reaches the critical value, the system control stability is close to the critical state.

In addition, through the simulation experiment, we can know that CAN network intervention did not significantly affect the stability of vehicle braking. Therefore, when CAN network communication environment is good, the network induced delay of CAN network has a little influence on the performance of the controller, which indicates that the ABS controller built in this research has strong robustness on a single road.

In the 80 s of last century, the researchers began to try to decompose the complex chassis control problem into a number of subcontrol systems and then use a mechanism to coordinate the dynamic relationship between the subsystems to meet the control requirements. Using the upper coordinated control for the integrated control architecture of multiple independent control units of the vehicle chassis can effectively adjust the collaborative work of the control units, avoid the conflict between the controllers, and make the vehicle obtain optimal running state.

The supervision mechanism is based on a hierarchical control principle, combined with fuzzy control logic to design a controller to supervise and coordinate ESP, AFS, and ARS. The target of the upper supervisory controller, according to the stability factor to judge vehicle steady state, is to redistribute the control weights of three subsystems and coordinate the output of each subcontroller.

The sensors necessary for many systems are designed as independent network nodes. The subcontrol systems adopt traditional point-to-point connection in the controllers, sensors, and executing agencies. Its object is to obtain satisfactory real-time performance and coordination.

\section{Conclusions}

In this paper, the vehicle chassis control system is taken as the application of CAN network. The target focuses on how the network affects the control system. The ABS, ASC, and IVC are simulated. The main research contents and conclusions are as follows.

According to the control theory of sliding mode, ESP and AFS subcontrollers are designed to track the ideal yaw rate. Based on the principle of hierarchical control and fuzzy control, a fuzzy controller is designed, which is used to monitor and coordinate the ESP, AFS, and ARS. And the IVC system is constructed with the upper supervisory controller and three subcontrol systems on the Simulink platform. Compared with the point-to-point connection, the system simulation of IVC-NCS shows that the control of the integrated control system has good performance.

According to the IVC based on the supervision mechanism, combined with the function of each subsystem, the network topology structure of IVC is proposed, and the IVC communication matrix based on CAN network communication is designed. With the common sensors and the subcontrollers as the CAN network independent nodes, the network induced delay and packet loss rate on the system control performance are studied by simulation. The simulation results show that the network does not lose frame, and even if the network traffic load is close to 1 , the network intervention of IVC can only show the very small change of the dynamic quality of the system. The network packet loss has a significant impact on the performance of the system control. When the packet loss rate is less than $30 \%$, only the system dynamic performance becomes worse, and the system stability does not change. When the packet loss rate is up to $50 \%$, the system control stability is close to the critical state, and the vehicle is unstable.

\section{Competing Interests}

The authors declare that they have no competing interests.

\section{Acknowledgments}

This study was funded by The Natural Science Foundation of Jiangsu Province (BK20130977). 


\section{References}

[1] T. Gordon, M. Howell, and F. Brandao, "Integrated control methodologies for road vehicles," Vehicle System Dynamics, vol. 40, no. 1-3, pp. 157-190, 2003.

[2] H. Chou and B. D’Andréa-Novel, "Global vehicle control using differential braking torques and active suspension forces," Vehicle System Dynamics, vol. 43, no. 4, pp. 261-284, 2005.

[3] C. B. Chu and W. W. Chen, "Vehicle chassis system based on layered coordinated control," Chinese Journal of Mechanical Engineering, vol. 44, no. 2, pp. 157-162, 2008.

[4] H. Zhu and W. W. Chen, "Active control of vehicle suspension and steering system based on strategy hierarchy," Chinese Journal of Agricultural Machinery, vol. 39, no. 10, pp. 1-6, 2008.

[5] M. J. L. Boada, B. L. Boada, A. Munoz, and V. Diaz, "Integrated control of front-wheel steering and front braking forces on the basis of fuzzy logic," Proceedings of the Institution of Mechanical Engineers, Part D: Journal of Automobile Engineering, vol. 220, no. 3, pp. 253-267, 2006.

[6] D. Li, X. Shen, and F. Yu, "Integrated vehicle chassis control with a main/servo-loop structure," International Journal of Automotive Technology, vol. 7, no. 7, pp. 803-812, 2006.

[7] E. J. Bedner and H. H. Chen, "A supervisory control to manage brakes and four-wheel-steer systems," SAE Paper 2004-01-1059, 2004.

[8] S. Chang and T. J. Gordon, "A flexible hierarchical model-based control methodology for vehicle active safety systems," Vehicle System Dynamics, vol. 46, supplement 1, pp. 63-75, 2008.

[9] N. Kelling, "The BRAKE project-centralized versus distributed redundancy for brake-by-wire systems," SAE Paper 2002-01-0266, SAE International, 2002.

[10] J. X. Wang, Research of Integrated Control System of Vehicle Chassis Based on Multi Agent, Southeast University, Nanjing, China, 2010.

[11] Y. Zhang, C. L. Yin, and J. W. Zhang, "A real time estimation method for the lateral velocity of the center of mass of the vehicle," Chinese Journal of Mechanical Engineering, vol. 44, no. 2, pp. 219-222, 2008.

[12] D. Crolla and Y. Fan, Vehicle Dynamics and Control, Chinese Communications Press, Beijing, China, 2003.

[13] H. B. Pacejka and E. Bakker, "Magic formula tyre model," Vehicle System Dynamics, vol. 21, no. 1, pp. 1-18, 1993.

[14] H. B. Pacejka and I. J. M. Besselink, "Magic formula tyre model with transient properties," Vehicle System Dynamics, vol. 27, supplement 1, pp. 234-249, 1997.

[15] K. H. Guo and L. Ren, "A unifield semi-empirical tire model with higher accuracy and less parameters," SAE Technical Paper Series 1999-01-0785, SAE International, 1999.

[16] H. Dugoff, P. S. Fancher, and L. Segal, “Tyre performance characteristics affecting vehicle response to steering and braking control inputs," Final Report, US National, 1969.

[17] W. Jinxiang and C. Nan, "Research on supervisory control based integrated chassis control framework and its simulation," Transactions of the Chinese Society of Agricultural Machinery, vol. 40, no. 9, pp. 1-6, 2009.

[18] S. Inagaki, I. Kushiro, and M. Yamamoto, "Analysis on vehicle stability in critical cornering using phase-plane method," in Proceedings of the International Symposium on Advanced Vehicle Control (AVEC '94), pp. 287-292, Tsukuba- Shi, Japan, 1994. 


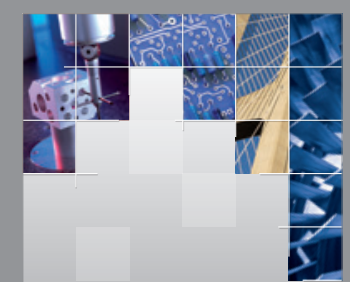

\section{Enfincering}
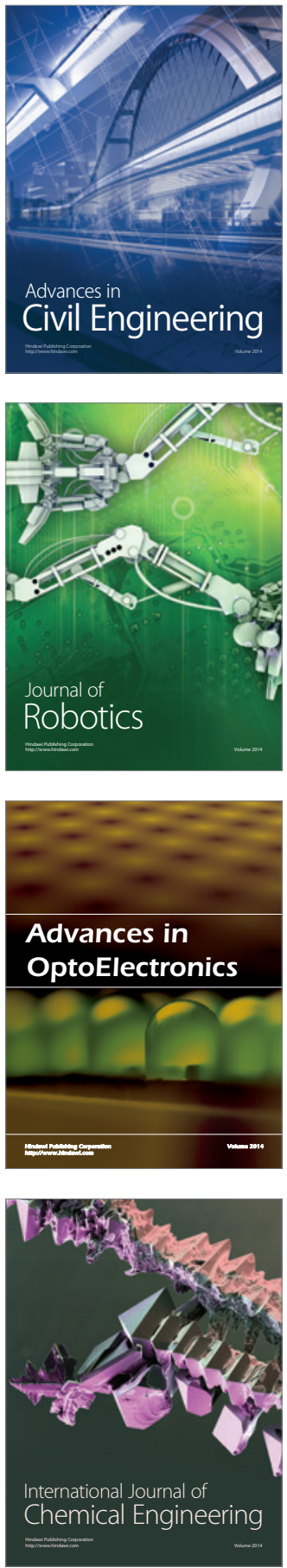

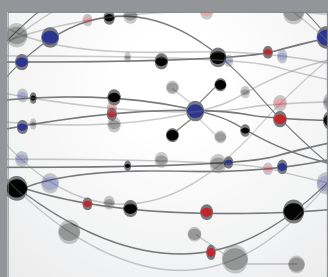

The Scientific World Journal

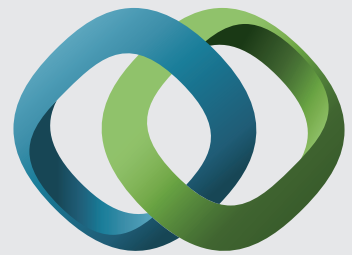

\section{Hindawi}

Submit your manuscripts at

http://www.hindawi.com
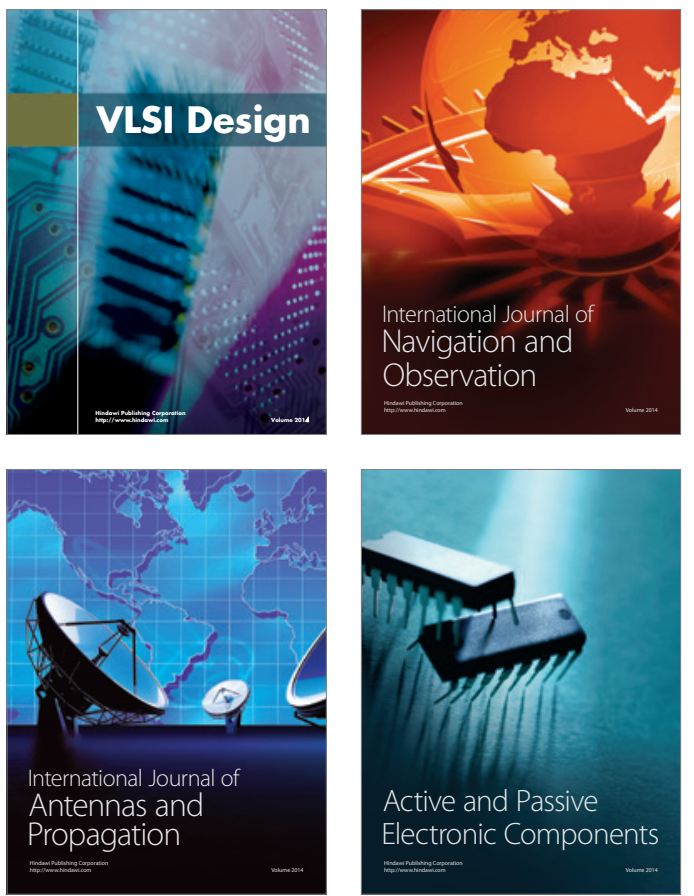
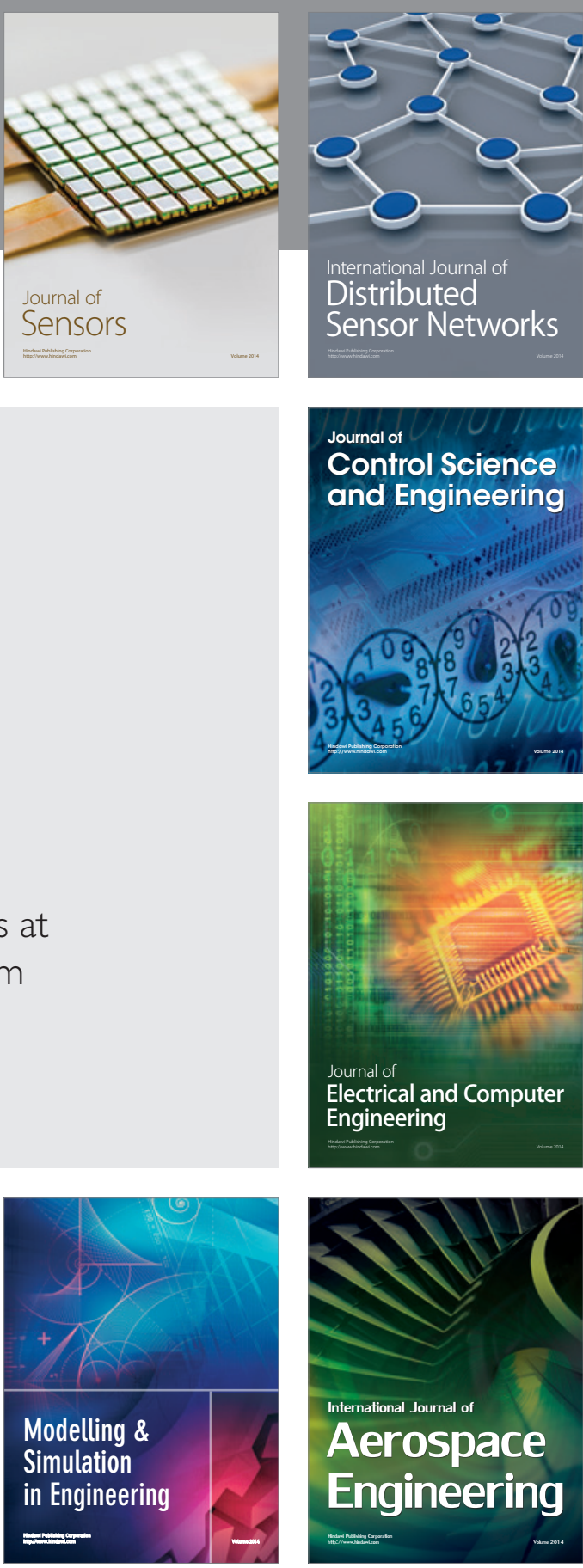

International Journal of

Distributed

Sensor Networks

Journal of

Control Science

and Engineering
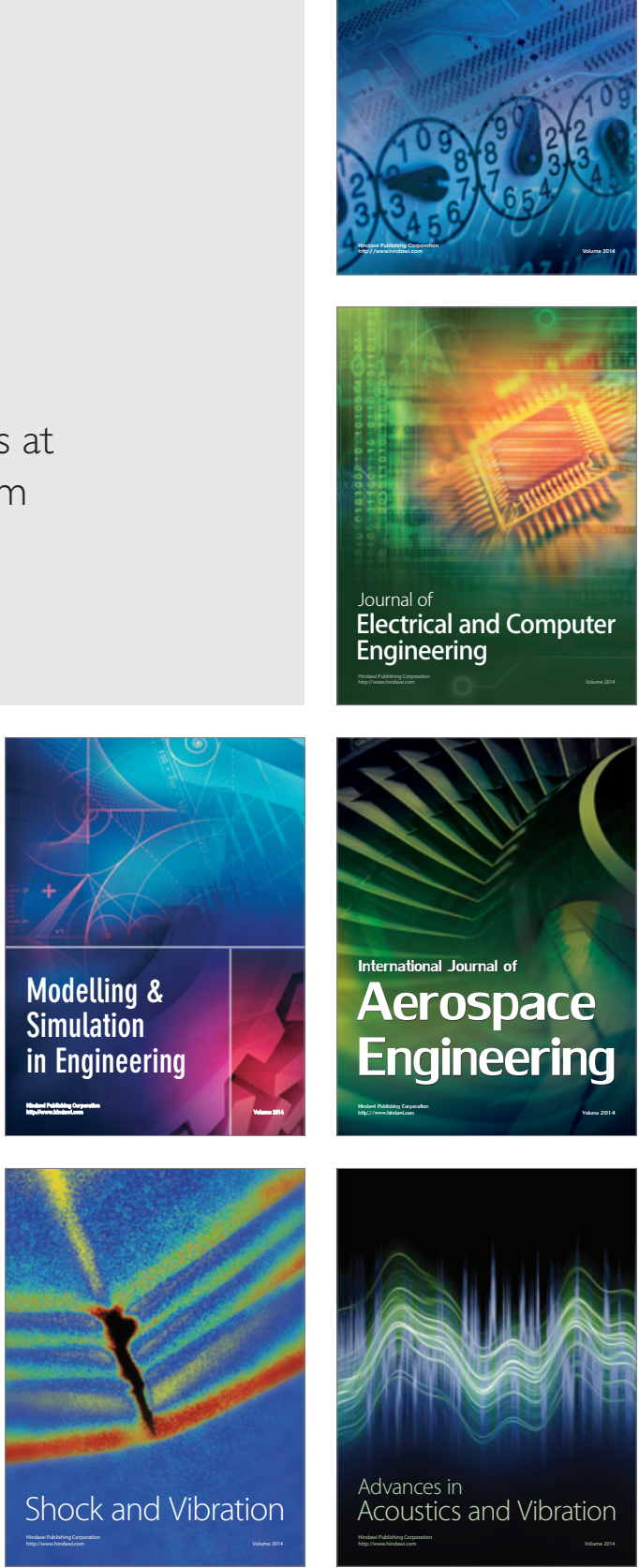